\title{
INFERENCIA ABDUCTIVA BASADA EN MODELOS. UNA RELACIÓN ENTRE LÓGICA Y COGNICIÓN
}

\author{
Alejandro Ramírez Figueroa \\ Departamento de Filosofía \\ Facultad de Filosofía y Humanidades \\ Universidad de Chile \\ alramire@uchile.cl
}

RESUMEN: La abducción ha desempeñado un papel principal en los enfoques filosóficos sobre la generación de nuevas hipótesis explicativas en las ciencias. Por su parte, desde el punto de vista de la ciencia cognitiva, el razonamiento basado en modelos es el principal instrumento para el pensamiento creativo en la ciencia. Esta segunda clase de razonamiento no se aplica a sentencias sino a objetos semánticos denominados modelos y no está determinada por reglas formales de inferencia. Este artículo sostiene la tesis de que la abducción, en su papel de descubridora, se ve reforzada por la "abducción basada en modelos", figura que no ha sido suficientemente analizada en la literatura actual sobre el tema.

PALABRAS CLAVE: razonamiento, descubrimiento, diagrama, hipótesis, conocimiento

SUMMARY: Abductive reasoning has played a central role in philosophical accounts of generation of new explanatory hypotheses in science. On the other hand, cognitive scientists have considered model-based reasoning as a main instrument for creative thinking in science. This latter kind of reasoning is applied not to sentences but to semantic objects called models, and is not governed by formal rules of inference. This paper holds the thesis that abductive inference, in its discovery role, is reinforced by what can be characterized as "model-based abduction", a notion which has not yet been sufficiently analyzed in the literature.

KEY WORDS: reasoning, discovery, diagram, hypotheses, knowledge

\section{Introducción}

Es notorio que algunas de las ideas centrales de las ciencias, como afirman por ejemplo Nersessian (1999, p. 7; 2002; 2008), Thagard (1993, cap. 4; 1978), Shelley (1996, p. 281), como las de inercia, selección natural, fuerza, gravitación, mercado, fósil, y otras, no pueden ser producto de generalizaciones de observaciones, ni de algoritmos, sean deductivos o inductivos, sino de cierta capacidad cognitiva en general oscura, difícil de describir, normalmente reconocida como generación de nuevas ideas, pero que la ciencia cognitiva ha logrado elucidar en sus aspectos centrales. La importancia epistemológica de la creatividad fue planteada por los empiristas lógicos, pero la solución que dieron al problema de cómo se generaban nuevas ideas teóricas no fue más allá de aludir a la inefable capacidad humana de inventar, de crear ideas nuevas, de "conjeturar felizmente". Afirmar 
esto casi equivale, sin embargo, a no dar ninguna solución en absoluto. Por otra parte, tampoco en los razonamientos de la vida cotidiana la producción de ideas nuevas quedaba resuelta sino mediante lo insondable y misterioso de las capacidades de la subjetividad humana para inventar.

Hubo que esperar hasta N.R. Hanson (1958, 1960, 1967), para que tal capacidad empezara a ser considerada, lo cual se hizo echando mano de la inferencia abductiva, concebida por C.S. Peirce justamente como aquel razonamiento capaz de inferir una hipótesis explicativa a partir de cierto hecho extraño. La teoría abductiva se convirtió, así, en el expediente de lo que se denominó a partir de allí una "lógica del descubrimiento", que se volvió un tema legítimo en filosofía de las ciencias y, hoy en día, en filosofía de la lógica y en ciencia cognitiva. La cuestión en disputa era claramente el estatus de "lógica" que tenía la abducción y la idea según la cual la "creación" humana no era un hecho susceptible de ser pensado con reglas. Así, la transformación hansoniana ha sido profunda: de no ser una cuestión epistemológica lícita, la generación de hipótesis y su naturaleza lógica se ha convertido en un problema central en las últimas cuatro décadas.

Si bien la inferencia abductiva puede dar cuenta de la generación de una nueva idea en la vida cotidiana o de una hipótesis científica, no parece, empero, ser hoy el único camino para teorizar al respecto. A partir de los denominados razonamientos basados en modelos, uno de cuyos principales impulsores fue Philip N. Johnson-Laird desde la década de 1980 (1988, 1991, 1995, 2005), y antes de él, Kenneth Craik (1967), en la ciencia cognitiva se ha arribado a otro esquema posible de inferencia para encarar el problema. JohnsonLaird concibió que en el razonamiento "natural", tanto en la calle como en la ciencia, parece que inferimos apelando no a enunciados y reglas formales explícitas sino creando y manejando ciertos objetos de carácter semántico a los que llamó "modelos mentales".

Desde el punto de vista de la psicología cognitiva, la cuestión se centra en el "razonamiento real", entendido éste como un hecho subjetivo, fáctico, que le acaece a un sujeto en cuanto sujeto psicológico real. Se trataría de entender cómo razonamos los seres humanos. Al respecto ha habido dos tendencias principales en ciencia cognitiva: la primera postula que razonamos gracias a que subjetivamente tenemos impresas reglas sintácticas que están en correspondencia con las reglas de la lógica; la otra tendencia afirma que eso no es posible, que no podemos tener interiorizadas reglas algunas, y que más bien apelamos a otros recursos, como son los modelos mentales, que pue- 
den corresponder a otros tipos de lógicas no clásicas. ${ }^{1}$ Esto podría conducir a pensar que es imposible conciliar lógica y cognición. Pero tal visión — se propone aquí- sólo es cierta en parte: en la parte que muestra que la lógica, como disciplina, es diferente de la psicología cognitiva. Pero ello, aunque obvio, no impide correlacionarlas. La lógica es central en las discusiones cognitivistas, en las que se trata de entender cómo opera el razonamiento, qué es lo que sucede cuando se deduce. Por ejemplo, según Johnson-Laird, lo que ocurre cuando cotidianamente, o en la ciencia, aplicamos un modus tollendo tollens es que creamos y manipulamos ciertos modelos mentales y razonamos con ellos. Por otra parte, hay intentos actuales en los que se trata de formalizar y dar una semántica al razonamiento basado en modelos (en adelante, RBM), como es el caso de los trabajos de Bharath Chandrasekaran (2006), Janice Glasgow y Andrew Malton (1999) o Keith Stenning et al. (2008).

Ahora bien, las relaciones entre lógica y cognición son aún difusas $\mathrm{y}$, al parecer, no se han instaurado todavía como un tema suficientemente perfilado para la filosofía de la lógica, de la ciencia y la epistemología. En el presente artículo se trata de establecer una relación específica circunscrita al problema de lo que se denominaba "lógica del descubrimiento". La tesis es que existe algo así como la abducción basada en modelos, forma inferencial que toma elementos del RBM y la abducción. Se plantea que el nexo entre ambos niveles es doble: por un lado, radica en que el RBM puede reforzar y complementar el razonamiento abductivo como base inferencial del descubrimiento. Por su parte, y a la inversa, el razonamiento basado en modelos parece tomar la forma de abducción y, especialmente, la de una inferencia al mejor modelo. Considerada solamente dentro de los márgenes proposicionales, la abducción se vuelve un recurso muy estrecho e insuficiente pues deja fuera elementos extralingüísticos que parecen intervenir en muchos razonamientos. A partir de las propuestas de algunos autores actuales, principalmente JohnsonLaird (1988, 1991, 1995, 2005), Shelley (1996) y Magnani (2001), se investigará, entonces, qué figura puede tomar una inferencia como la abducción basada en modelos. En dicha figura "modelo-abductiva" intervienen tanto elementos lógico-formales como otros provenientes de la ciencia cognitiva, en especial de la psicología cognitiva.

${ }^{1}$ Al respecto puede verse, por ejemplo, Johnson-Laird y Byrne 2000, Tabossi et al. 1999, Garnham y Oakhill 1994. Piaget fue uno de los impulsores de la tesis de la lógica mental, la cual poseería, incluso, una base biológica (1981). 
En la sección 2 se exponen las ideas centrales de lo que es el RBM. En la sección 3 se analiza si se puede afirmar que en Peirce se encontraba ya alguna expresión de una abducción basada en modelos. En la sección 4 se examina cómo la abducción basada en modelos responde a la condición de deducción en reversa. En la sección 5 se aborda la idea de abducción basada en modelos en Johnson-Laird, Magnani y Shelley y, finalmente, en la última sección se propone la idea de una inferencia al mejor modelo.

\section{Deducción y razonamiento basado en modelos}

El pensamiento inferencial ha sido entendido de ordinario como un proceso principalmente sintáctico, que consiste sobre todo en transitar sistemática y reguladamente de unos enunciados a otro final, que se apoya en los primeros. ${ }^{2}$ Si bien tal visión analítica es el fundamento de las ciencias formales, desde hace décadas se viene pensando en que ello no agota toda visión posible sobre el razonar. La idea de razonamiento parece ampliarse; en ciertas situaciones, no en todas, por supuesto, los sujetos parecen acudir a otros formatos distintos de los enunciados y las reglas de la lógica. Uno de ellos, el que aquí se analiza, son los modelos, entidades de tipo semántico que pueden ser físicos o mentales.

2.1 .

Consideremos este ejemplo simple: un arquitecto proyecta una estructura básica cuyo volumen se inscribe en un cubo; tal figura puede ser descrita por un conjunto pequeño de enunciados, $E_{1} \ldots E_{n}$. Supongamos que a ese cubo se le agrega un cubo menor junto a una de sus caras, y ambos quedan conectados por una escalera. Describir lo anterior mediante un conjunto de enunciados $E_{1} \ldots E_{n}$, donde $n$, ahora, es mucho mayor, puede ser algo ya engorroso. Vale más la pena construir un modelo, físico o mental, de tal situación (B. Tversky et al. 1999; Tversky 2005). Pero, ¿qué sucede si tal arquitecto pregunta si entre ambos cubos hay suficiente espacio como para transitar de uno al otro sin toparse con la escalera? ¿O si la visión desde el interior del cubo menor se verá obstaculizada hacia el cubo mayor por la posición de la escalera?, y otras preguntas por el estilo. ¿Cómo obtener una conclusión respecto de cuál debe ser la posición adecuada de tal escalera y sus dimensiones? Si, además, por necesidades del

\footnotetext{
${ }^{2}$ Sobre esto hay dos visiones distintas pero complementarias en lógica: la semántica y la sintáctica.
} 
proyecto entre manos, el segundo volumen no puede estar al mismo nivel que el primero sino, digamos, a un tercio de su altura, ¿qué implica ello para las preguntas anteriores? Está claro que ante ello es posible enumerar un conjunto de enunciados descriptores de la situación. Pero también parece manifiesto que, en ese caso, recurrir al conjunto $E_{1} \ldots E_{n}$ de enunciados es algo demasiado dificultoso. Si consideramos ahora no dos simples cubos unidos por una escalera, sino algo mucho más complejo, el recurso proposicional quedará por completo fuera de lugar y será materialmente imposible de manejar, salvo por medios computacionales. Lo que hace el arquitecto en cuestión es construir primero una situación mental que pueda traducirse después en un modelo físico, lo cual dependerá de la dificultad del asunto, tal que lo rote, lo manipule, y, mediante tal recurso, pueda inspeccionar lo que sucede y "extraer" una conclusión plausible. Ha razonado, y tal vez lo haya hecho válidamente, esto es deductivamente, no mediante la aplicación de alguna regla formal explícita, sino construyendo un modelo e inspeccionándolo. Se supone que lo que concluye de tal modelo imaginado o materializado será válido para el volumen real que va a construir.

\section{2}

Otro ejemplo, casi canónico en estos temas, es el razonamiento específicamente deductivo, de carácter visual, a partir de ciertos enunciados en calidad de premisas. Si (i) $A$ está a la izquierda de $B$, y (ii) $B$ a la izquierda de $C$, y (iii) además se sabe que un triángulo está debajo de $A$, con los correspondientes significados de "estar debajo de" y "estar a la izquierda de", responder en qué relación espacial están $C$ y el triángulo requiere construir un modelo de la "situación" descrita por el enunciado, no del enunciado propiamente tal. Dicho modelo, físico o mental, debe cumplir ciertas condiciones, pero la principal es la representatividad entre modelo y lo modelado. Así, tenemos:

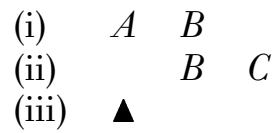

Ante la formación del modelo puede "verse", inspeccionando (manipulando) el modelo, inmediatamente lo que se deduce, esto es, la posición de $C$ respecto del triángulo. Allí está la transitividad lógica sin que esté explícita dicha regla. Es más, teniendo a la vista la con- 
figuración de modelos de la situación enunciada en (i)-(ii)-(iii), se pueden responder otras preguntas o deducir otras conclusiones.

Así, el RBM está compuesto por la construcción e inspección posterior de un modelo de la situación descrita, tal que haya una analogía entre la estructura de la situación y la del modelo, para así concluir, por simple inspección, que $\boldsymbol{\Delta}$ está a la izquierda y por debajo de $C$. Y también a la izquierda y por debajo de $B$. En este caso, la información inicial tiene formato proposicional, pero esto se soluciona mediante un modelo. En otros casos, la información inicial puede ser visual y se construyen modelos con base en ella. El caso del arquitecto se asemeja más a este último.

Características centrales del modelo son, pues, las siguientes:

(a) Es un objeto representacional icónico, en general no sentencial.

(b) La relación representacional se da por semejanza estructural o funcional entre el modelo y lo modelado.

(c) El modelo no es una imagen mental de algo; si lo fuera, no sería un modelo, sino una copia. El modelo selecciona ciertos rasgos que interesan.

(d) Por lo tanto, la modelación es una cuestión pragmática, que se hace para algo específico.

De modo que el RBM consiste en:

(1) La formación de un modelo de una situación.

(2) La manipulación, inspección y observación del modelo formado.

(3) La conclusión directa sobre el modelo; esto significa que el sujeto no lee en el modelo tal como se lee un letrero, sino que "extrae" del modelo una información que estaba implícita en las premisas. Lo hace mediante una inspección, porque la conclusión se revela en el modelo mismo. Ese sentido de "extraer" es lo que da sentido inferencial al RBM.

(4) La suposición de que lo inferido del modelo vale para lo modelado. En consecuencia, lo que hace el RBM, tanto si es válido como si no lo es, es conservar, en la conclusión, la representabilidad de lo observado en los modelos. Se puede denominar a esto último, "principio de preservación de la representación".

(5) El RBM puede configurarse como un razonamiento exclusivamente visual, en cuyo caso las premisas están dadas en una escena; o puede ser heterogéneo, cuando se mezclan enunciados con modelos. 
(6) La base de esto es que el modelo no es un mero instrumento auxiliar del razonamiento, sino una parte integrante de él (véase, al respecto, Barwise y Etchemendy 1996, p. 5).

Johnson-Laird (1995) propuso su teoría del razonamiento sobre la base de modelos, en específico modelos mentales, principalmente para dar cuenta de la deducción, aunque no de manera exclusiva. Definió la idea de modelo mental con los siguientes tres principios: a) iconicidad; b) posibilidad; y c) verdad. Los tres — se puede agregar — están coordinados por otro principio, que es central: la representabilidad. El modelo es un artefacto abstracto que representa situaciones de un objeto, persona, sistema, sean reales o ficticios. Si no hay relación de representación entre el modelo, físico o mental, y lo representado, no puede hablarse de un modelo propiamente.

\section{3 .}

Los tres principios anteriores pueden quedar ejemplificados en un caso de deducción silogística, que es el tipo de razonamiento en el cual Johnson-Laird se centró prioritariamente. Su interés principal fue, pues, la deducción. Tomemos este silogismo partiendo de los ejemplos propuestos por él: ${ }^{3}$ (i) Ningún $A$ es $B$; (ii) Todos los $C$ son $B$; (iii) por lo tanto, ningún $A$ es $C$. Johnson-Laird afirma que hay una forma de exteriorizar los modelos mentales en tablas como las siguientes:

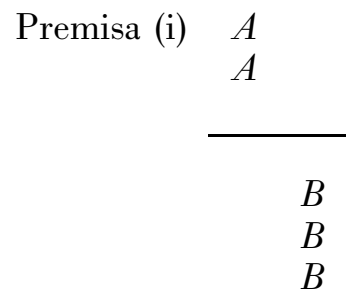

Premisa (ii), se agrega sobre la anterior:

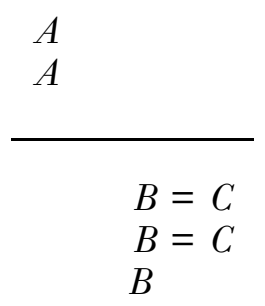

${ }^{3}$ Johnson-Laird 1991, p. 478, 2005, y véase también 1988. 
La función de la línea horizontal es separar los diferentes ejemplares o tokens (no se trata de la línea lógica de inferencia). Así, lo que se lee en el segundo modelo, que unifica ambas premisas y que se construye superponiéndolo al primero, es lo siguiente: que todos los $B$ son $C$ y que ningún $C$ es $A$, que están en el lado superior de la línea horizontal. Se ve entonces de inmediato que ningún $A$ es $C$, ya que están, cada uno, a un lado de la línea sin tocarse. La existencia del último token $B$ aislado, sin $C$, indica que podría haber algún $B$ que no sea $C$, pues lo que afirma la segunda premisa es que todos los $C$ son $B$, no que todos los $B$ sean $C$. (Todos los chilenos son sudamericanos pero no a la inversa.) Lo notorio de este caso, que no trata de relaciones espaciales como el caso 2.2, es que el razonamiento se establece sin aplicar las reglas explícitas del silogismo: se lleva a cabo mediante una inspección visual-mental graficable de la disposición de los tokens. ${ }^{4}$

El primer principio es el de iconicidad. Si bien Johnson-Laird ha establecido estos principios para modelos mentales, pueden ser extrapolados a todo modelo, esto es, a modelos de tipo físico, como son los diagramas, los mapas, las construcciones volumétricas, todos ellos modelos visuales. Un modelo es icónico porque contiene un token para cada referente del discurso, como los enunciados de las premisas, o, también, para los referentes reales que han de ser modelados. Así, $A \ldots B$ es un modelo de " $A$ está a la izquierda de $B$ ", con dos tokens, $A$ y $B$. Pero el modelo tiene propiedades que deben corresponder con el referente o lo modelado; y hay relaciones en el modelo que deben corresponder con las relaciones del referente. Todo este conjunto hace que el modelo sea, a decir de Johnson-Laird, un "análogo estructural" del referente. En otros términos: "El modelo mental tiene una estructura que corresponde con la estructura conocida de lo que él representa" (Johnson-Laird 2005, p. 187). El modelo es, entonces, una representación pictórica del mundo, no es una representación lingüística.

${ }^{4}$ La teoría del razonamiento basado en modelos de Johnson-Laird es mucho más compleja de lo que muestra este ejemplo, pero éste basta para los propósitos aquí planteados. Pueden verse, para tal efecto, Johnson-Laird 2005, 1988; y JohnsonLaird y Byrne 2000. La teoría de los modelos mentales del autor requiere otros pasos; en algunos casos puede hacer falta construir más de un modelo. Además, una vez obtenida la conclusión, ésta tiene un carácter supuesto y debe someterse a la búsqueda de modelos que sirvan de contraejemplo, de forma que modelando las premisas se concluya un enunciado contradictorio con respecto de la primera conclusión. Si ello ocurriera, los primeros modelos eran errados. Esto se basa en el principio de validez lógica: de verdades no puedem concluirse falsedades. Para una crítica a esta teoría, véase Asti 2008, e incluso Johnson-Laird 1991. 
El segundo principio es el de posibilidad y asegura simplemente que cada modelo representa una posibilidad específica: un modelo representa una situación específica; no cabe hablar de modelos universales. En este punto surge un problema no fácil de solucionar y, aun cuando no es el tema aquí, conviene al menos tenerlo a la vista. Se trata de la posible distinción que pueda haber entre la idea de modelo como se la ha descrito aquí y los diagramas de Venn-Euler. Una cosa es cierta y es que el concepto de modelo, no obstante su profusa presencia hoy en temas de filosofía de las ciencias, lógica, epistemología y psicología cognitiva, no posee una figura consensuada y única, y seguramente no puede tenerla. Así, por ejemplo, la idea de modelo en el RBM no guarda relación con la teoría de modelos en lógica. ${ }^{5}$ Johnson-Laird defiende más bien la idea de que un modelo no corresponde completamente con los diagramas de Venn y, de allí podríamos afirmar que el RBM no corresponde, entonces, con la lógica diagramática y la lógica heterogénea, aunque hay una indudable familiaridad entre ambos campos y coincidencias en varios aspectos. ${ }^{6}$ Johnson-Laird (1991, p. 478) afirma que la distinción consiste, justamente, en que los modelos son representaciones de un trozo del mundo y de situaciones y así, por lo tanto, son específicos; mientras que un diagrama de Venn posee universalidad. Pero debe añadirse el hecho de que los diagramas de Venn no pueden representar enunciados de segundo orden, como "algunos de los $A$ son todos $\operatorname{los} B$ ". Con todo, la diferencia no parece tan clara. Mucho de lo que Allwein, Barwise y Etchemendy muestran como razonamiento diagramático corresponde muy de cerca con el RBM. Tal vez se pueda decir que el asunto radica en la pragmática; un modelo, en el RBM, posee siempre un sentido representacional; se modela para algo y por ello el modelo representa un trozo de mundo, aunque éste sea ficticio. En lógica diagramática, en cambio, el diagrama puede perfectamente no representar nada, ni situación, ni persona, ni estructura, ni función. Hay algo más que decir al respecto: como hemos visto, el modelo guarda una analogía estructural con el trozo de mundo, pero en el mero diagrama tal representación puede ser sólo convencional, tal como la luz roja del semáforo representa la prohibición de avanzar.

El tercer principio es el de verdad. Un sujeto, al construir un modelo mental de una situación, sólo retiene las posibilidades verda-

\footnotetext{
${ }^{5}$ Puede verse al respecto Giere 1999, que compara el modelo en el RBM y en lógica; el primero es representacional y el segundo es instancial.

${ }^{6}$ Véanse Allwein y Barwise 1996 en general, y, en particular, Barwise y Etchemendy 1996.
} 
deras. Johnson-Laird lo ejemplifica así: supongamos " $A$ o $B$ pero no ambos". El modelo "explícito" puede tener la siguiente figura:

$$
\begin{array}{cc}
A & \neg B \\
\neg A & B
\end{array}
$$

Lo anterior corresponde con las tablas de verdad de tal disyunción exclusiva. Sin embargo, el modelo mental sólo representa lo que se afirma, que $A$ o $B$, esto es:

\section{$A$}

$B$

En la primera línea no cabe representar no $B$, pues no se lo está afirmando. y lo mismo ocurre con la segunda línea. No se representa no $B$ porque es lo falso, ni no $A$ porque también es lo falso.

Finalmente hay que recalcar que un modelo en el RBM no es una imagen mental ni una copia física de algo; no es una copia de una situación pues el modelo selecciona rasgos. El mapa que modela un territorio no reproduce todos los rasgos del territorio; sería imposible y, sobre todo, inútil y sin sentido que lo hiciese. Si el modelo copiara o reprodujera todos los rasgos, no sería un modelo.

La deducción puede entonces ser representada como el RBM. ${ }^{7}$

3. Abducción basada en modelos: ¿tuvo su origen en Peirce?

El razonamiento basado en modelos no es, según lo visto en la sección precedente, una alternativa a la deducción, sino que la deducción puede basarse en modelos. Por lo tanto, y aun cuando solamente Lorenzo Magnani y, en menor medida. el mismo Johnson-Laird lo explicitan en forma clara, puede afirmarse que también es posible la abducción basada en modelos.

Si Peirce concibió la abducción como la inferencia que parte de hechos y concluye en una hipótesis que los explica, en un camino incierto y, desde luego, lógicamente no válido, pero creador y sugerente, aquí se postula que tal papel de la abducción como descubridora

${ }^{7}$ Otros autores que exploran actualmente la cuestión de la deducción basada en modelos son, por ejemplo, Chandrasekaran (2006), Tabossi et al. (1999), y Glasgow (1999), quien proporciona una semántica para la deduccion sobre la base de matrices (arrays). 
se ve reforzado y ampliado por el RBM, en la forma específica de una abducción basada en modelos. En otros términos, el RBM permitiría ampliar, enriquecer y fortalecer el sentido de la abducción al incorporar otros formatos de razonamiento, diferentes de los enunciados. Ahora bien, ¿es posible encontrar ya en Peirce una forma abductiva de RBM? ¿Postuló Peirce esos otros formatos? Peirce pensó el razonamiento en general no sólo en términos enunciativos, sino también visuales. He allí su teoría de los gráficos existenciales, y su tesis de que todo razonamiento es en realidad icónico. Debe elucidarse, entonces, si tales ideas constituyen una forma de razonamiento basado en modelos, tal como se ha definido actualmente.

La abducción, para Peirce, es una estructura inferencial de pleno derecho, diferenciable de la deducción y la inducción, aunque, según él, es la más débil de las tres. Comparte con la inducción la característica de ser "ampliativa", lo que las diferencia de la deducción; pero la abducción es más débil que la inducción, porque sus premisas prestan menos apoyo a la conclusión. Aun así su función es especial, ya que es la única estructura lógica que puede conducir a una idea nueva: si la deducción prueba y la inducción generaliza, dice más de lo mismo, la abducción genera. Peirce planteó dos formulaciones lógicas de la inferencia abductiva: la primera, llamada por el autor "hipótesis", es de 1878 y se puede formalizar proposicionalmente de la siguiente manera: $(B \wedge(A \rightarrow B)) \rightarrow A$, lo que, en términos estrictamente lógicos, es inválido, pues se trata de la falacia de la afirmación del consecuente $(C P, 2.623)$. $B$ significa un hecho "sorprendente" que se ha de explicar (el resultado, de acuerdo con Peirce), el condicional $(A \rightarrow B)$, una teoría de base o conocimiento ya adquirido desde donde se abduce (regla, en términos de Peirce), y $A$ es la hipótesis abducida (el caso según Peirce) que explica $B$. Una hipótesis no se abduce sólo desde el hecho sorprendente que se va a explicar, sino desde cierta información previa, sea ésta un conocimiento ordinario si se trata de un razonamiento en la vida cotidiana, o de una teoría científica contrastada positivamente, si se trata de una ciencia. Cabe hacer notar que la sola teoría de base no basta para abducir; se requiere, además, que esté en conjunción con los hechos que pretende explicar. A tal información ya aceptada se la denomina aquí, en general, "teoría de base". Esta formulación contiene una característica que ha conducido a los autores actuales a formular la estructura lógica de la abducción como una deducción en reversa más condiciones adecuadas (por ejemplo, Aliseda 2006, Flach 2000). 
Lo anterior significa que la hipótesis $A$ es abducida (Flach usa el símbolo $K)$ si dicha hipótesis en conjunción con el condicional $A \rightarrow B$ implican el que se quiere explicar, $B$. En términos de reglas, en el nivel metalingüístico: $(B, C) K A$ syss $C, A=B$. Esta formulación permite hoy el manejo de la abducción en términos de algoritmos computacionales. Esta condición general de reversibilidad $T, H K h$ syss $T, h=H$, requiere, a su vez, condiciones formales, epistémicas y pragmáticas, como la minimalidad o simplicidad de la hipótesis abducida; consistencia entre hipótesis y teoría de base; y explicación, esto es no puede deducirse el hecho que se intenta explicar solamente a partir de la teoría de base, sin la participación de la hipótesis: $T \neq h$ y $h \neq T .^{8}$

La segunda formulación que ofrece Pierce $(C P, 5.189)$ posee esta estructura: (i) Se observa cierto hecho $H$ sorprendente; (ii) si $A$ fuese verdadera, $H$ sería algo corriente, (iii) "por lo tanto, hay razones para sospechar que $A$ es verdadera". Dadas estas dos formulaciones, es posible indagar ahora si la abducción basada en modelos estaba ya implicada de alguna manera en el pensamiento de Peirce.

Un vestigio del RBM lo encontramos en Peirce $(C P, 5.162)$ enunciado así: "Todo razonamiento necesario, sin excepción, es diagramático. Esto es, construimos un ícono de nuestro estado hipotético de cosas y procedemos a observarlo. Esta observación nos conduce a sospechar que algo es verdadero, lo cual puede o no puede ser formulado con precisión." Por razonamiento necesario hay que entender aquí deducción. La formulación de Peirce alude al razonamiento diagramático, mas, en su generalidad, podría también interpretarse como RBM. En efecto, en tal expresión están al menos presentes dos elementos del RBM, según lo expresado en la sección 2: ante una situación se construye un elemento que no es sentencial, que tiene una capacidad de representar tal situación, lo cual hace el modelo. En segundo lugar, lo que se hace, una vez que se tiene el ícono formado, es observarlo, lo cual es justamente parte principal del RBM según Johnson-Laird, esto es, la manipulación del modelo para poder inferir algo a partir de él. Así, aunque Peirce se refiere en este pasaje a su propuesta de un razonamiento diagramático, parece haber una coincidencia básica con lo que se denomina hoy razonamiento basado en modelos.

Peirce expone el siguiente razonamiento: " $A$ es un caballo bayo. Por tanto, $A$ es un caballo", lo que es válido. Peirce pregunta, ¿cómo sabemos que es válido? La respuesta es:

${ }^{8}$ Esto se puede ver en detalle en Aliseda 2006, p. 71; 1998; 2004. 
Probablemente replicaríamos que imaginaríamos un caballo bayo y contemplando la imagen veríamos que es un caballo. Pero esto sólo se aplica a una imagen simple. ¿Cuán grande era la imagen que representa ese caballo? ¿Sería lo mismo con un caballo de diferente tamaño? ¿Cuán viejo era el caballo representado? ¿Tenía la cola cortada? ¿Sería lo mismo si el caballo sufriera de vértigo y, si así fuese, sería lo mismo si fuera cualquier otra enfermedad que padeciera el caballo? Estamos absolutamente seguros de que ninguna de estas circunstancias afectaría en absoluto este asunto. (CP, 5.164)

De este pasaje pueden extraerse algunos de los elementos del RBM: la realización del argumento por medio de la apelación a otro elemento, distinto de una sentencia, una imagen; observación directa en esa imagen y lectura en ella de una respuesta; selección de los elementos de la imagen que son pertinentes para saber si se trata de un caballo: para ello no se requiere contar con todas las características del objeto. ${ }^{9}$

Thagard y Shelley (1997) afirman que Peirce no trató explícitamente la cuestión de una "abducción visual", a pesar de haber creado una lógica diagramática. No obstante lo anterior, de lo expuesto parece posible concluir que Peirce al menos barruntó que la abducción puede hacerse sobre modelos.

\section{Abducción basada en modelos y deducción reversa}

Abducir con modelos parece responder al esquema de la deducción en reversa más condiciones adecuadas. Volvamos a un ejemplo visuoespacial sencillo: (i) Sabemos que $\boldsymbol{\Delta}$ está a la izquierda de $B$ y nada más puede estar a la izquierda de $B$; (iii) observamos el hecho de que $\boldsymbol{\Delta}$ está a la izquierda de $\bullet$, ¿cómo explicamos tal localización en función de considerar la localización relativa de $B$ respecto de $\bullet$ ? Se asume que el asunto está restringido por las relaciones únicas: "estar a la izquierda de" y "estar a la derecha de", y por ninguna otra. Además, (i) corresponde a lo que, en teoría de la abducción, se denomina teoría o conocimiento de base; (iii) corresponde, por su parte, al hecho que se quiere explicar mediante la abducción.

El problema abductivo consiste en responder esta interrogante: ¿qué debemos hipotetizar en (ii) acerca de la ubicación de $B$ tal que (ii) más la premisa (i) impliquen la ubicación indicada en (iii)?

${ }^{9}$ En 2.619 y ss., Peirce expone un ejemplo que se acerca mucho al RBM; en él, ante la observación de restos de un papel escrito, el sujeto construye una imagen de una situación donde puede inferir (abducir) de la "forma" del papel una hipótesis sobre quién es el autor, tal que explique la ubicación y la rotura del papel. 
Consideraremos el modelo $M$ como conformado por elementos (letras y figuras) y por las líneas, en este caso tres, (i)-(ii)-(iii), que se organizan en una estructura de líneas y columnas $(a, b, c, d, e)$. Así, el modelo $M$ es el siguiente:

$M: \quad a \quad b \quad c \quad d \quad e$
(i)
(ii)
(iii)

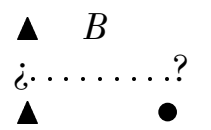
( $T$, teoría de base)
(pregunta abductiva)
( $h$, hecho)

Modelos $M^{\prime}$ : análisis sobre cinco abducibles posibles para la línea (ii):

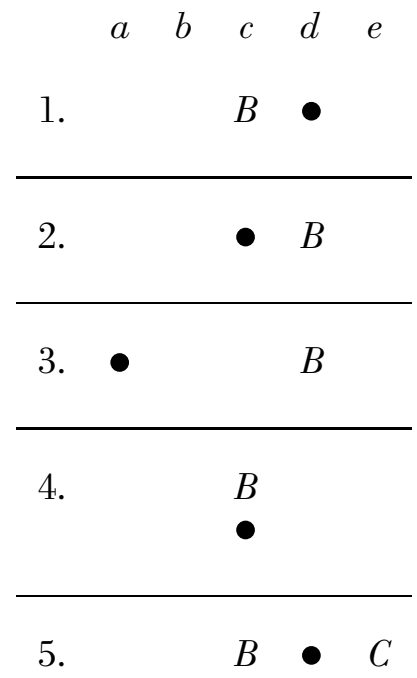

Analicemos estas cinco alternativas. Lo primero que debemos considerar es que los modelos propuestos no son los únicos posibles: en principio, podría proponerse un número infinitos de modelos supuestamente abducibles con sólo variar la ubicación relativa de cada elemento en su respectiva línea. Los modelos 3 y 4 no son abducibles, puesto que no cumplen con la deducción en reversa. En los casos 2 y 3 , - queda a la izquierda de $B$ (columna $a$ ), lo que está prohibido por la condición inicial, aunque el caso 2 cumple con la deducción en reversa: ambos deben ser eliminados como abducibles. En el caso 4, "estar encima de" no está permitido tampoco, por lo que dicho caso no puede participar de la derivación de (iii). Los modelos 1 y 5 son 
los únicos abducibles, pues cumplen con la condición de deducción en reversa y con las condiciones de: $T \not h$ y $h \neq T$. Debe notarse que si bien 5 es abducible, pareciera tener menos simplicidad que 1 , puesto que 5 tiene información redundante. Abordaré este aspecto en la última sección de este artículo, al tratar la ABM como inferencia al mejor modelo.

Cabe hacer una última observación sobre este ejemplo. La abducción sobre la base de modelos se verifica por inspección visual de la construcción previa de un modelo, constituido por las tres líneas (i), (ii) y (iii). Pero también son modelos cada una de las líneas abducidas en (ii), por lo que la ABM, para modelar (i), (ii) y (iii), debe modelar a su vez la línea (ii) abducida.

\section{Abducción basada en modelos: posturas en desarrollo}

La idea de una abducción basada en modelos es un tema aún en desarrollo. La mayor parte de los autores se centran en la deducción. Algunos de ellos, como Nancy Nersessian (1999, 2002, 2008), incluso desconfían de que exista realmente algo como la abducción. Conviene, pues, examinar algunas posturas que conciben que sí es posible la abducción no proposicional.

Johnson-Laird (2005) presta atención a otras formas inferenciales, como el razonamiento probabilístico y, en alguna medida, a lo que aquí llamamos abducción basada en modelos. Su idea central es que la abducción depende principalmente de conocimientos causales, de modo que, dado un efecto, hay más de una causa posible que lo explique. La abducción es un razonamiento del efecto a la causa posible. Equivale a la creación de explicaciones (Johnson-Laird 2005, p. 201).

El esquema abductivo de Johnson-Laird no apela al recurso de la deducción en reversa, aunque no lo excluye. Dicho instrumento no es, en realidad, de carácter cognitivo, que es el punto de vista del autor. La abducción se da en una situación en la que un sujeto, ante un determinado problema de inconsistencia, modela, esto es, crea un modelo de una situación causal, que explique esa inconsistencia. El autor ejemplifica así este proceso de modelación: (i) Si el gatillo se aprieta, la pistola dispara; (ii) el gatillo se aprieta y la pistola no dispara. En esa situación hay una inconsistencia. Lo que el sujeto requiere es abducir una hipótesis que explique tal inconsistencia, o, específicamente, que ofrezca una explicación del enunciado (ii) dado el enunciado (i). La cuestión es que, como toda abducción, se requiere apelar a un conocimiento previo, o teoría de base, puesto 
que la abducción no es una adivinación, una "conjetura feliz", como afirmaban los empiristas lógicos. La hipótesis se genera desde una base. En este ejemplo, los sujetos abductores saben, por ejemplo, que: a) una pistola sin balas no funciona; que b) el percutor en mal estado no produce el disparo; que c) la bala en mal estado tampoco, etc., etc. Entonces, el sujeto imagina (en este caso el modelo no es visuoespacioal) un modelo en el que se produce, por ejemplo, la siguiente situación causal:

\section{$M:$ Sin balas — presiona el gatillo — no hay disparo}

Este modelo que el sujeto produce permite abducir, junto con los conocimientos preexistentes, que, por ejemplo: "alguien quitó las balas". En este caso, el RBM se configura con los siguientes elementos:

- Situación (i) y (ii)

- Conocimiento de base, a) y b)

- Construcción del modelo $M$

- Hipótesis abducida que explica (i) y (ii)

Yu (2002) aborda la cuestión específicamente desde la filosofía de las ciencias, en una mirada al mismo tiempo ontológica: hay similaridad en el mundo, y ello permite el RBM. Se ha considerado central - afirma $\mathrm{Yu}$ - que dos aspectos del razonamiento científico sean la prueba y el descubrimiento. Es en lo segundo donde es pertinente la abducción basada en modelos, a lo que denomina "razonamiento creativo": "Necesitamos extender nuestra idea de razonamiento científico para incluir razonamientos complejos y creativos" (2002, p. 276). El modelo mental — recalca $\mathrm{Yu}$ $^{10}$ no sólo es una representación icónica de una situación real: también puede serlo de cosas que no existen, como ocurre con el modelo de la partícula en mecánica clásica. Allí, lo que se modela es un objeto inexistente, esto es, sólo conjeturado. Se supone una entidad denominada partícula a la que se le atribuyen determinadas propiedades. Es interesante este planteamiento, ya que previene a la tesis de RBM de los avances de algún tipo de realismo.

\footnotetext{
${ }^{10} \mathrm{Yu}$ trabaja específicamente sobre la idea de modelo mental de Johnson-Laird, aun cuando los ejemplos que expone muchas veces están elaborados a partir de modelos físicos o visuales.
} 
La representación es una relación entre estructuras: entre la estructura del modelo y la estructura supuesta del objeto inexistente. Esto es algo central en la comprensión de la ciencia.

El modelo construido de una entidad física idealizada a la que se denomina partícula permitió razonar abductivamente hacia la formulación hipotética de propiedades del comportamiento de los cuerpos, en su calidad de objetos sometidos a fuerzas. ¿Cómo se descubrió o supuso la partícula, si acaso se pudiera decir así? ¿@O las ondas, o los fotones? Por inducción, no. En consecuencia, Yu no ve como alternativa más que la conjetura basada en modelos; en otros términos, la abducción basada en modelos. Aquí sigue a Johnson-Laird, entre otros, y afirma que la abducción es el razonamiento que va desde el efecto hasta la posible causa. El esquema que ofrece para ello no es sino una versión de la abducción de Peirce: 1) ley de experiencia correlativa $L ; 2$ ) si una hipótesis teórica es verdadera, $L$ es explicada; 3) tenemos razones para reconocer $H$ como verdadera. La diferencia con la formulación de Peirce es que, en vez de un hecho extraño por explicar, aparece una ley $L$ en necesidad de explicación, lo cual en la ciencia suele ser el caso. Dado que las entidades teóricas son inobservables, el científico sólo puede abducir lo que podría ser el caso partiendo de un análogo sobre el cual el científico está bien informado y traspasando la información del análogo para proponer propiedades y características de la nueva ley, la ley $L$ abducida. "El facsímil o proyecto, creado de esa manera, es el modelo mental" ( $\mathrm{Yu}$ 2002, p. 281). Y sigue: "Aunque el modelo mental es creado, entonces, en referencia al análogo, representa el mecanismo de causa y efecto envuelto en el fenómeno bajo investigación."

La abducción basada en modelos mentales descansa en un aspecto ontológico: la similaridad entre objetos, similitudes que pueden ser de cualidades pero también de funciones. Hay similaridad en el mundo y ello es lo que permite la analogía, y la analogía estaría en la base de la construcción de modelos. El concepto de similaridad es central en la comprensión de la naturaleza. Justamente la ciencia ha mostrado que la similitud recorre toda la naturaleza, en todos sus niveles. Todas las plantas son, desde cierto punto de vista, similares entre sí; todos los mamíferos lo son; todos los átomos lo son; todas las personas lo son. Sin embargo, ello significa que, en la naturaleza, la similtud no permita otra cosa, ni la identidad ni lo diferente: "las cosas en la naturaleza no son absolutamente las mismas, ni son absolutamente diferentes" (Yu 2002, p. 285). Si la modelación es la selección de aspectos similares entre el modelo y lo modelado, la existencia de 
la similtud es lo que permite el razonamiento basado en modelos y, específicamente, la abducción basada en modelos.

Tal vez sean Lorenzo Magnani y Cameron Shelley los autores que más han desarrollado hasta el día de hoy la idea de una abducción basada en modelos, respecto de su naturaleza y sus proyecciones. Magnani en un sentido epistemológico más abstracto, y Shelley de manera más específica, en relación con el razonamiento en arqueología. Magnani y Belli 2006 y Magnani $2001^{11}$ proponen una tipología básica, no exhaustiva, de los problemas que piden ser solucionados por medio de una abducción visual (Magnani restringe aquí su estudio al razonamiento visual, donde los modelos son visuales-espaciales). Dichos problemas tienen que ver con asuntos del siguiente tipo: a) explicar la ausencia de un objeto; b) explicar por qué un objeto está en una determinada posición; c) reconocer que un objeto posee una determinada función (ser una herramienta, por ejemplo). Todos éstos, y otros similares, piden un razonamiento de tipo abductivo y, dado que el asunto se plantea en el ámbito visual-espacial, el razonamiento tenderá a realizarse también por medio de modelos visuales.

Magnani plantea así la cuestión: "¿Cómo puede un razonamiento visual llevar a cabo una explicación? Para responder a esto es necesario mostrar cómo la abducción visual puede ser relevante para la generación de hipótesis, esto es, cómo una explicación basada en imágenes puede resolver un problema dado en una imagen inicial" (Magnani 2001, p. 107). La abducción basada en modelos se sintetiza en el siguiente procedimiento:

\section{Imagen inicial.}

II. Reconocimiento de una cuestión problemática, en la imagen inicial.

III. Construcción de una imagen hipotética que explique el problema implicado en la imagen inicial.

Dado este proceso, entonces, afirma Magnani: "la imagen formada adquiere el estatus de una hipótesis en el proceso abductivo" (2001, p. 107), y agrega: "la generación de una nueva imagen hipotética puede ser considerada como el resultado de una abducción creativa" (2001, p. 108). Un ejemplo mostrado por el autor expresa el caso b) de su tipología: explicar la posición relativa de un objeto en una situación dada.

${ }^{11}$ Magnani 2001 desarrolla sus ideas de la abducción visual en consonancia con la teoría de las matrices (arrays) propuesta por Glasgow y Malton 1999. 
I. Observamos vidrios rotos sobre el piso de una habitación, hojas cerca de la mesa desparramadas por el suelo, y abierta la ventana de la habitación. Ésa es la imagen inicial.

II. La imagen inicial es anómala, de acuerdo con la experiencia y los conocimientos del sujeto, y se compara con la situación ordenada en la memoria de largo plazo.

III. Abducción: si somos capaces de construir una imagen ficticia que represente una situación posible que explique lo ocurrido y en la cual, por ejemplo, sea verosímil un robo, o que el viento haya roto la ventana $\mathrm{u}$ otra, estamos construyendo una explicación.

De acuerdo con el RBM, tal explicación se lee inmediatamente en la imagen, en el modelo, sin estar mediada por representaciones enunciativas. Por otra parte, se cumple la condición del RBM según la cual el modelo no es necesariamente una copia: es manifiesto que el modelo de la situación anómala no tiene que detenerse en rasgos que no sean atingentes o que no se juzguen como tales. Si la imagen modelada no ofrece una explicación admisible de lo ocurrido en I, debe buscarse otra.

La tipología ofrecida por Magnani posee aplicaciones claras en algunas disciplinas, como es el caso de la arqueología. Reconocer la función de cierto objeto o explicar la posición de un objeto son cuestiones relevantes en arqueología. Shelley (1996), Thagard (1978) y Thagard y Shelley (1997) han explorado la abducción basada en modelos en esa disciplina científica. En términos generales podemos decir que determinar la función posible de cierto objeto $X$ encontrado en cierto contexto dado pasa por construir un modelo de la situación en que se visualice cierta función de ese objeto $X$; la construcción de dicho modelo visual requiere conocimiento arqueológico previo por parte del sujeto que modeliza. Si en ese modelo visual el objeto $X$ cumple una función que encaja con aquellos conocimientos que el arqueólogo posee sobre esos contextos, podemos abducir la plausibilidad de que $X$ sea una herramienta de tipo $Y$ más que de tipo $Z$, dado que esa herramienta y no otra es consistente con la teoría de base de la arqueología.

Magnani ha ampliado actualmente su primer análisis de la naturaleza de la abducción (Magnani 2001) y lo ha dirigido hacia una comprensión amplia de la naturaleza pensante humana, e incluso de la naturaleza animal en general (Magnani 2009). Proponer una hipótesis, afirma él, es uno de los principales actos cognitivos; por esa razón, la abducción no sólo es un tipo de inferencia, sino además un mecanismo fundamental del pensamiento. Magnani incorpora temas centrales 
de la ciencia cognitiva, como son las ideas de cognición distribuida, situada y extendida (Clark 2007). Pero, en lo que tiene más interés para este artículo, desarrolla, siguiendo lo ya esbozado en su primer libro, la idea de que la abducción es un proceso cognitivo central de naturaleza híbrida, multimodal, que añade no sólo las sentencias sino también otros formatos, como son los elementos sensoriomotores, las simulaciones, las emociones, el pensamiento analógico y las visualizaciones. Magnani profundiza su tesis según la cual la abducción es un razonamiento que actúa no sólo en formato inferencial, sino también modélico. Interesantes son los casos analizados acerca de los razonamientos con modelos visuales en ciencias. El concepto de tangente a una curva, por ejemplo, en un análisis primero se representa como un punto común entre la curva y la tangente. Alcanzando "nuevas representaciones internas", "nuevas representaciones mentales" (Magnani 2009 , p. 80), éstas producen un nuevo conocimiento: vemos que lo que parecía un punto debería analizarse ahora como una relación entre puntos, rectas y áreas. El modelo o representación interna de esa situación, específicamente su "manipulación", nos presenta una nueva situación hipotética acerca de la tangente. ${ }^{12}$ Magnani relaciona, pues, abducción y cognición de una manera que abarca muchos aspectos del pensamiento. Uno de ellos es, sin duda, el razonamiento basado en modelos de orden abductivo. Un punto que tal vez pueda indicarse como distintivo respecto del enfoque que se aborda en este artículo tiene que ver justamente con el concepto mismo de modelo. En el trabajo de Magnani no se termina de aclarar dicha idea, que parece abarcar cualquier gráfico o diagrama, lo cual haría que el RBM fuera equivalente al recurso metodológico de los círculos de Venn o a la inferencia heterogénea, por ejemplo, cuestión que aquí tratamos de diferenciar.

Shelley (1996, p. 280), por su parte, propone una tipología similar a la de Magnani, pero recogida específicamente de problemas arqueológicos:

1. Abducción relacionada con el reconocimiento de un objeto con cierta función.

2. Abducción que tiene que ver con las formas de los objetos.

${ }^{12}$ En Magnani (2009, pp. 139 y ss.) también pueden verse ejemplos de lo que el autor denomina "abducción explicativa", en la que se da cuenta, mediante representaciones geométricas, de algo no entendido o "sorprendente". Ejemplifica asimismo la abducción creativa, que permite arribar a nuevos conocimientos. Magnani interpreta el descubrimiento de la geometría no euclidiana como un caso de ello. 
3. Abducción que se relaciona con la determinación de la estructura de un objeto.

El reconocimiento de objetos en arqueología es, en todo caso, cualitativamente distinto - muestra Shelley - del que Peirce asignó como función a la abducción. En el ejemplo de Peirce, el que reconozcamos o percibamos un objeto como negro es la mejor explicación de que es negro realmente (1996, p. 280). Sin embargo, reconocer un objeto en arqueología como esto o lo otro es algo más complejo. Si bien, recalca Shelley, la representación modélica no es necesaria, en muchos casos es el mejor modo de comprender lo que sucede en el razonar arqueológico. De ordinario, un objeto encontrado, digamos un fósil, no es algo que inmediatamente sea reconocible como tal, mucho menos su función, por varias razones. El objeto se encuentra mezclado con la roca, confundido en color y textura; además, casi siempre, aparece fragmentado en trozos, lo cual dificulta mucho su reconocimiento. Ángulos de encaje, dimensiones y posiciones conforman un rompecabezas que puede ser infinitamente complejo. La construcción de un modelo visual de la situación, de su posible forma, puede ser determinante. Es difícil explicar todo ello mediante un reconocimiento perceptual, como se mencionó antes en referencia a Peirce; se requiere más. Es más plausible que tal reconocimiento apele a la modelización de una situación donde encaje el objeto, modelo cuya manipulación permita abducir que se trata de un fósil y no de otra cosa. La tarea de reconocimiento es más compleja de lo que aparenta. La abducción basada en modelos es una forma de entender cuál sea el modo de razonamiento implicado allí.

Shelley afirma que existe una correlación inversa entre la necesidad de aplicar la abducción basada en modelos y las tareas de reconocimiento arqueológico. La necesidad de construir modelos visuales y de razonar abductivamente - se puede decir - se incrementa a medida que la tarea es menos familiar. Si la tarea de reconocer un objeto pasa por situaciones similares, el arqueólogo puede razonar inmediatamente y reconocer el objeto, por asociación con lo ya conocido, sin necesidad de abducción basada en modelos. En disciplinas como la arqueología, en la que la investigación visual es parte primaria de la práctica de la disciplina, la abducción basada en modelos parece tener una relevancia especial.

\section{6. ¿Inferencia al mejor modelo?}

Si en el razonamiento abductivo proposicional la inferencia abduce un enunciado con carácter de explicación hipotética, ¿qué se abduce real- 
mente en la abducción basada en modelos? Si se considera lo antes expuesto, tal interrogante parece tener dos respuestas posibles que hacen complejo el asunto. La primera es que lo que se abduce es un enunciado como producto de la manipulación del modelo. Ejemplo de ello es el caso arqueológico de identificación de un objeto como una herramienta, en la que el modelo visual de una situación permite concluir que, digamos, "El objeto $X$ es una herramienta con función cortante". En tal dirección también podemos incluir el razonamiento abductivo con modelos mentales en Johnson-Laird. Sin embargo, interesa aquí sobremanera la otra alternativa: lo que se abduce en una abducción con modelos es el modelo mismo. Proponemos aquí que en la abducción basada en modelos lo que se abduce es determinado modelo, que el modelo producido en el razonamiento cumple varias funciones, como fomentar comprensión, dar explicación del problema y ser, finalmente, la hipótesis generada.

Volvamos una vez más al ejemplo de razonamiento visuoespacial de la sección 2. En este ejemplo se puede ver de manera muy sencilla lo que ya se adelantó en dicha sección: que, para una determinada situación, parece siempre haber más de un modelo que la represente. Esto último conduce naturalmente a la cuestión de cuál es el mejor modelo. Una respuesta general será que el mejor modelo es aquel que facilite en mayor medida el obtener la abducción, en este caso, o la conclusión en general. Se puede pensar al respecto en algo así como un principio de facilitación o simplificación del razonamiento.

El modelo de la situación abductiva en cuestión era el siguiente:
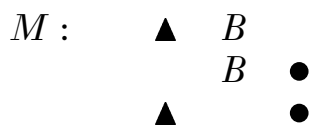

Es manifiesto que tal modelo $M$ de la situación completa no es el único posible. En términos muy sencillos bastaría considerar el siguiente modelo $M^{\prime}$ para constatar que la disposición de ambos modelos es la misma, que podemos razonar de la misma forma y la conclusión se extrae, en rigor, de ambas:

$M^{\prime}$

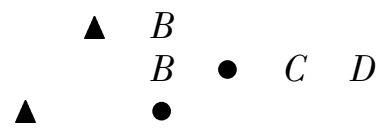

Pero también resulta evidente que, para efectos de la lectura, de la inspección visual del modelo no resultan $M$ y $M^{\prime}$ equivalentes. Y lo 
que interesa es justamente que el modelo "muestre" inmediatamente la situación. Si bien, en este caso, la conclusión puede seguir siendo que el triángulo está a la izquierda del círculo, la cuestión es que cabe preguntarse cuál es el mejor modelo, si $M$ o $M^{\prime}$. De acuerdo con el principio establecido de facilitación del razonamiento, no parece discutible que el mejor modelo es $M$, puesto que es más fácil leer la conclusión en $M$ que en $M^{\prime}$. También puede decirse que $M$ es más simple que $M^{\prime}$, criterio también importante. Esto es así porque $M^{\prime}$ introduce elementos redundantes, $C, D$, que no cumplen ningún papel en la deducción de la tercera línea y, sobre todo, introducen una distracción visual en la lectura de la ubicación relativa de los elementos del modelo. En otros términos, la estructura del modelo se ve sobrecargada de información irrelevante. Esto se relaciona también con el hecho de que un modelo tiene la función de hacer comprender una situación, de servir de explicación, por lo que es válido pensar en que haya mejores o peores modelos que cumplan esa función. Debe elegirse, pues, el mejor modelo posible.

Así, en analogía con la inferencia a la mejor explicación, parece que se puede plantear una inferencia al mejor modelo, cuya estructura simple sería:

Situación problemática $S$

Si $M$ es Modelo de $S$, hay comprensión de $S$

Modelo $M^{\prime}$ de $S$

$M$ es mejor que $M^{\prime}$

\section{$M$ explica la situación $S$}

Hay que considerar que, desde Gilbert Harman (1965), muchos autores, la mayoría en realidad, con algunas excepciones como Aliseda (1998), Niiniluoto (1999), o Thagard (1993), consideran la inferencia a la mejor explicación como una forma abductiva o, más aún, como dos estructuras idénticas. El mismo Hanson, que redescubrió a Peirce y estableció el tema de la generación de hipótesis como un tema en filosofía de las ciencias, lo consideró así. No es el caso discutir tal identificación, pero, de seguirla, tendremos que la inferencia al mejor modelo que hemos esbozado corresponde a una abducción sobre la base de modelos, que es lo que se quería mostrar. 
Cuando James D. Watson y Francis Crick (Giere et al. 2006, cap. 2; y Giere 1999) construyen el modelo de una triple hélice molecular, cuando de la manipulación de éste no llegan a los resultados buscados, cuando producen un segundo modelo, de doble cadena de moléculas y de la manipulación de éste sí logran los resultados esperados, puede decirse que los investigadores han producido un modelo que es el que explica el problema: que lo abducido es el modelo mismo. En tal sentido, hay una inferencia al mejor modelo. Cuando ante el problema de identificar la función de un objeto se logra un modelo tal que ese objeto aparece claramente cumpliendo la función de herramienta cortante, como lo hemos visto antes, lo que se abduce es ese modelo, pues es ese modelo el que comporta explicación. Está claro, entonces, que muchos modelos pueden ser abducidos ante una situación dada, y alguno debe poder ser considerado como el que mejor explica. Ése es el que se adoptará.

La concepción estándar del RBM, la de Johnson-Laird, no parece considerar esta última alternativa. El RBM se percibe solamente como aquel que construye un modelo, lo manipula y de allí concluye, deduce o abduce. Pero que el modelo sea, él mismo, lo abducido, como aquí lo hemos presentado, no es algo que se suela considerar parte de la cuestión. Con todo, hemos podido mostrar de qué manera ambos sentidos de la ABM son complementarios.

\section{Conclusiones}

No existe en la actualidad una teoría general o unificada sobre los razonamientos basados en modelos, y, en consecuencia, tampoco sobre la abducción basada en modelos. Pero las diversas posturas y aproximaciones que existen acerca de ella suponen ya la aceptación de que es posible y plausiblemente interesante la inferencia abductiva que vaya más allá del razonamiento sobre la base de reglas. Ello es lo que se ha querido mostrar, en primer lugar.

Una segunda cuestión es que resulta defendible la tesis de que la $\mathrm{ABM}$ es un reforzamiento de la abducción en su papel de descubridora. Si bien el razonar con modelos no es algo que tenga carácter necesario, dado que la mayoría de las veces es reemplazable por enunciados, en determinadas situaciones argumentativas resulta útil, y puede ser el mejor camino para explicar cómo razonamos en realidad. Existen situaciones complejas en las que un modelo permite lo que los enunciados dificultan. Así, la tarea del descubrir - tradicionalmente desde Peirce-Hanson atribuida a la abducciónpuede verse ahora mejor explicada con una herramienta al menos 
complementaria, como es la figura de la ABM, en sus dos posibilidades, como manipulación de modelos y como inferencia al mejor modelo. ${ }^{13}$

\section{BIBLIOGRAFÍA}

Aliseda, A., 2006, Abductive Reasoning, Kluwer, Dordrecht.

— 2 2004, "Logics in Scientific Discovery", Foundations of Science, vol. 9, no. 3, pp. 339-363.

— 1998 , "La abducción como cambio epistémico: C.S. Peirce y las teorías epistémicas en inteligencia artificial", Analogía Filosófica, vol. 12, pp. 125-144.

Allwein, G. y J. Barwise, 1996, Logical Reasoning with Diagrams, Oxford University Press, Oxford/Nueva York.

Asti, C., 2008, Escenarios argumentativos, Educando, Buenos Aires.

Barwise, J. y J. Etchemendy, 1996, "Visual Information and Valid Reasoning", en Allwein y Barwise 1996, pp. 3-25.

Chandrasekaran, B., 2006, "Diagrams as Physical Models to Assist in Reasoning”, en Magnani 2006, pp. 285-300.

Clark, A., 2007, "Curing Cognitive Hiccups: A Defense of Extended Mind", The Journal of Philosophy, vol. 104, no. 4, pp. 163-192.

Craik, K., 1967, The Nature of Explanation, Cambridge University Press, Cambridge.

Flach, P., 2000, "On the Logic of Hypothesis Generation", en P. Flach y A. Kakas (comps.), Abduction and Induction, Kluwer, Dordrecht, 2000, pp. 89-105.

Garnham, A. y J. Oakhill, 1994, Thinking and Reasoning, Blackwell, Oxford.

Giere, R., 1999, "Using Models to Represent Reality", en Magnani et al. 1999, pp. 41-57.

Giere R., J. Bickle y R. Mauldin, 2006, Understanding Scientific Reasoning, Thomson/Wadsworth, Belmont.

Glasgow, J. y A. Malton, 1999, "A Semantics for Model-Based Spatial Reasoning", en Rickheit y Habel, 1999, pp. 259-297.

Hanson, N.R., 1967, "An Anatomy of Discovery", The Journal of Philosophy, vol. 64, no. 11, pp. 321-352.

_ 1960, "More on The Logic of Discovery", The Journal of Philosophy, vol. 57, no. 6, pp. 182-188.

- 1958, "The Logic of Discovery", The Journal of Philosophy, vol. 55, no. 25, pp. 1073-1089.

Harman, G., 1965, "The Inference to the Best Explanation", The Philosophical Review, vol. 24, no. 1, pp. 88-95.

${ }^{13}$ Este artículo se ha desarrollado como parte del proyecto de investigación patrocinado y financiado por Fondecyt-Chile No. 1095020, en los años 2009-2010. 
Holyoak, K. y R. Morrison (comps.), 2005, The Cambridge Handbook of Thinking and Reasoning, Cambridge University Press,

Johnson-Laird, P.N., 2005, "Mental Models and Thought", en Holyoak y Morrison 2005, pp. 185-208.

—_, 1995 (1983), Mental Models, Harvard University Press, Cambridge, Mass.

—_, 1991, "Mental Models", en M. Posner (comp.), Foundations of Cognitive Science, MIT Press, Cambridge, Mass., 1991, pp. 469-499.

—_ 1988, "Deduction", The Computer and the Mind, Harvard University Press, Cambridge, Mass.

Johnson-Laird, P.N. y R. Byrne, 2000, "The Cognitive Science of Deduction", en P. Thagard (comp.), Mind Readings, MIT Press, Cambridge, Mass., 2000, pp. 29-58.

Magnani, L., 2009, Abductive Cognition. The Epistemological and EcoCognitive Dimensions of Hypothetical Reasoning, Springer-Verlag, Berlín/Heisenberg.

—-2001, Abduction, Reason and Science, Kluwer, Dordrecht.

(comp.), 2006, Model Based Reasoning in Science and Engineering, College Publications, Londres.

Magnani, L. y E. Belli, 2006, “Agent-Based Abduction”, en Magnani 2006, pp. 415-439.

Magnani, L. et al. (comps.), 1999, Model Based Reasoning in Scientific Discovery, Kluwer, NuevaYork/Dordrecht.

Nersessian, N., 2008, Creative Scientific Concepts, MIT Press, Cambridge, Mass.

—_ 2002, "The Cognitive Basis of Model-Based Reasoning in Science", en P. Carruthers, et al. (comps.), The Cognitive Basis of Science, Cambridge University Press, Cambridge, 2002, pp. 135-153.

__, 1999, Model-Based Reasoning in Conceptual Change", en Magnani et al. 1999, pp. 5-22.

Niiniluoto, I., 1999, "Defending Abduction”, Philosophy of Science, vol. 66, no. 3, pp. S436-S451.

Peirce, C.S., 1965, The Collected Papers of Charles S. Peirce [CP], ed. C. Hartshorne y P. Weiss, Harvard University Press, Cambridge, Mass.

Piaget, J., 1981, Biología y conocimiento, trad. Francisco González Aramburu, Siglo XXI, Madrid/México.

Rickheit, G. y C. Habel (comps.), 1999, Mental Models in Discourse Processing and Reasoning, Elsevier, Amsterdam.

Shelley, C., 1996, "Visual Abductive Reasoning in Archaeology", Philosophy of Science, vol. 63, no. 2, pp. 278-301.

Stenning, K. y M. van Lambalgen, 2008, Human Reasoning and Cognitive Science, MIT Press, Cambridge, Mass.

Tabossi, P., V. Bell, y P.N. Johnson-Laird, 1999, " Mental Models in Deductive, Modal and Probabilistic Reasoning", en Rickheit y Habel 1999, pp. 299-331. 
Thagard, P., 1993, Computacional Philosophy of Science, MIT Press, Cambridge, Mass.

- 1978, "The Best Explanation: Criteria for Theory Choice", The Journal of Philosophy, vol. 75 no. 2, pp. 76-92.

Thagard, P. y C. Shelley, 1997, "Abductive Reasoning: Logic, Visual Thinking, and Coherence", en M.L. Dalla Chiara et al. (comps.), Logic and Scientific Methods, Kluwer, Dordrecht, 1997, pp. 413-427.

Tversky, B., 2005, "Visuospatial Reasoning", en Holyoak y Morrison 2005, pp. 00-00.

Tversky, B., J. Kim y A. Cohen, 1999, "Mental Models of Spatial Relations and Transformations from Language", en Rickheit y Habel 1999, pp. 209-240.

Yu, Q., 2002, "Model Based Reasoning and Similarity in the World", en L. Magnani et al. (comps.), Model-Based Reasoning: Science, Technology, Values, Kluwer, Nueva York, 2002, pp. 275-285.

Recibido el 3 de noviembre de 2010; revisado el 5 de julio de 2011; aceptado el 9 de noviembre de 2011. 